ARTICLE

\title{
Tailoring atomic diffusion for in situ fabrication of different heterostructures
}

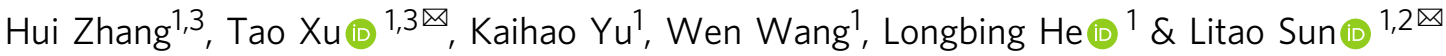

\begin{abstract}
Atomic diffusion has been recognized as a particularly powerful tool in the synthesis of heterostructures. However, controlled atomic diffusion is very difficult to achieve in the fabrication of individual nanostructures. Here, an electrically driven in situ solid-solid diffusion reaction inside a TEM is reported for the controlled fabrication of two different heteronanostructures in the Ag-Te system. Remarkably, the morphology and structure of the asformed heterostructures are strongly dependent on the path of atomic diffusion. Our experiments revealed that the surface diffusion of $\mathrm{Te}$ atoms to $\mathrm{Ag}$ nanowires leads to a coreshell structure, while the bulk diffusion of $\mathrm{Ag}$ atoms give rise to a $\mathrm{Ag}_{2} \mathrm{Te}-\mathrm{Te}$ segmented heterostructure. Heat released by Joule heating caused the surface diffusion process to be replaced by bulk diffusion and thereby determined the structure of the final product. Our experimental results provide an insight into solid-state diffusion reactions under an electric field and also propose a new process for the fabrication of complex nanostructures.
\end{abstract}

\footnotetext{
${ }^{1}$ SEU-FEI Nano-Pico Center, Key Laboratory of MEMS of Ministry of Education, School of Electronic Science and Engineering, Southeast University, Nanjing, China. ${ }^{2}$ Center for Advanced Materials and Manufacture, Joint Research Institute of Southeast University and Monash University, Suzhou, China.

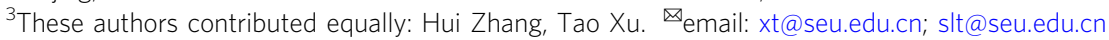


etero-nanostructures comprising multiple functional components integrated together can combine the advantages of their individual components and often achieve synergistic properties beyond the functionality of a single component $t^{1-3}$. In this context, one-dimensional (1D) heterostructures have attracted significant attention because of the unique physical and chemical properties arising from heterointerfaces, which enable their applications in various fields, such as energy storage ${ }^{4-6}$, catalysis ${ }^{7-9}$, biomedical sensing ${ }^{10,11}$, optoelectronic devices, etc. 1D hetero-nanostructures can be categorized into core-shell structures and segmented binary structures in terms of the morphology ${ }^{12,13}$, which are used in different applications. Generally, the morphology of the heterostructures is heavily determined by the synthetic method. Over the past decades, a variety of methods have been developed to synthesize 1D heterostructures, which fall into three main categories: heterogeneous nucleation and growth ${ }^{14}$, diffusion and deposition $^{15}$, and melt-casting and electrospinning ${ }^{16}$. Among these methods, diffusion methods are particularly attractive in view of their relative simplicity. The formation of new materials or new phases by diffusion methods including simple heterogeneous atomic diffusion, Kirkendall effect, ion exchange, or phase transformation in solid-state can be used to prepare 1D heterostructures with improved interface and morphology control. Great efforts have been made to achieve controlled diffusion and heterogeneous structure conversion. For example, Chen et al. $^{17}$ developed a solution-phase in situ diffusion growth method with heating treatment to fabricate $\mathrm{Fe}(\mathrm{OH})_{3} @ \alpha-\mathrm{MoO}_{3}$ core-shell nanorods. Resasco et al. ${ }^{18}$ demonstrated that controlled and uniform doping in a heterogeneous material (Ta-doped $\mathrm{TiO}_{2}$ ) can be achieved using a solid-state diffusion approach based on atomic layer deposition. Zhang et al. ${ }^{19}$ reported an electrically driven in situ cation exchange reaction for the fabrication of $\mathrm{CdS}-\mathrm{Cu}_{2} \mathrm{~S}$ core-shell structured nanowires. These results indicate that the diffusion method is very promising for the fabrication of heterostructures. However, faced with the need to minimize the size of electronic and photonic devices, researchers have made great efforts to develop efficient and controllable synthetic methods for individual nanomaterials for functional applications. To date, the tailored diffusion of atoms and ions and the mechanism at the nanoscale of solid-state diffusion reactions are bottlenecks in the efficient synthesis of $1 \mathrm{D}$ heterostructures for highly integrated nanodevices.

Herein, we conduct in situ tailored diffusion experiments inside a transmission electron microscope (TEM) system while applying a voltage bias. Using this method, it is possible to efficiently control atom diffusion to selectively fabricate either a core-shell nanostructure or a segmented binary heteronanostructure in the Ag-Te nanowire (NW) system. Migration under an electric field is the main driving force that promotes atomic diffusion ${ }^{20-22}$, which in turn, controls the direction of diffusion of $\mathrm{Ag}$ or $\mathrm{Te}$ atoms. Local temperature caused by Joule heating may strongly affect the mode of diffusion (bulk diffusion or surface diffusion) of particles owing to the different diffusion activation energies and ultimately results in the formation of different structures, including core-shell nanostructures resulted from surface diffusion and binary hetero-nanostructures resulted from bulk diffusion. Hence, our method makes it possible to fabricate complex nanostructures with controlled specific structures.

\section{Results}

In situ experiment. Ag and Te NWs (Supplementary Fig. 1) used in this work were synthesized by a previously reported solvothermal chemical approach ${ }^{23,24}$. Electrically driven fabrications of $1 \mathrm{D}$ heterostructures were conducted inside a TEM (FEI Titan 80-300 operated at $300 \mathrm{kV}$ ) equipped with a TEM and a scanning tunneling microscopy (TEM-STM) holder. As illustrated in Fig. 1a, Te NWs were supported on a TEM grid, and the Ag NW mounted on an STM probe was controlled to contact an individual Te NW, followed by the formation of different 1D heterostructure by applying a bias voltage.

It is rather remarkable that the morphology and structure of the as-formed heterostructures are tailored by the direction of applied voltage. Under the application of positive voltage to the Ag NW against the Te NW for a few minutes, as shown in Fig. 1b, a significant number of Te atoms migrate into the $\mathrm{Ag} \mathrm{NW}$ and eventually form an $\mathrm{Ag}_{2} \mathrm{Te}-\mathrm{Ag}$ core-shell structure. In contrast, if the bias voltage is reversed, an $\mathrm{Ag}_{2} \mathrm{Te}$-Te binary heterostructure is formed ultimately (Fig. 1c). Because of the formation of a semiconducting $\mathrm{Ag}_{2}$ Te layer, a Schottky-type contact is formed at the $\mathrm{Ag}_{2} \mathrm{Te}-\mathrm{Ag}$ interface and the current-voltage $(I-V)$ curve transforms from line shape to an S-shaped curve (Fig. 1d, e).

Formation of core-shell structures. In order to understand the mechanisms behind the transformation, we first observed how an $\mathrm{Ag} \mathrm{NW}$ reacts to form a core-shell structure. Figure $2 \mathrm{a}-\mathrm{f}$ present a typical series of TEM images showing the shape evolution of an Ag NW under a positive voltage of $0.3 \mathrm{~V}$ (Supplementary Movie 1). This dynamic process can be divided into two stages. In the first stage, small hill-like protrusions are formed on the Ag NW surface (red arrows in Fig. 2b, c), whereas no obvious changes are seen on the Te wire. Next, the hillocks grow and fuse into larger hills, eventually forming a clear core-shell structure (Fig. 2d-f). After the reaction, high-resolution transmission electron microscope (HRTEM) images and corresponding fast
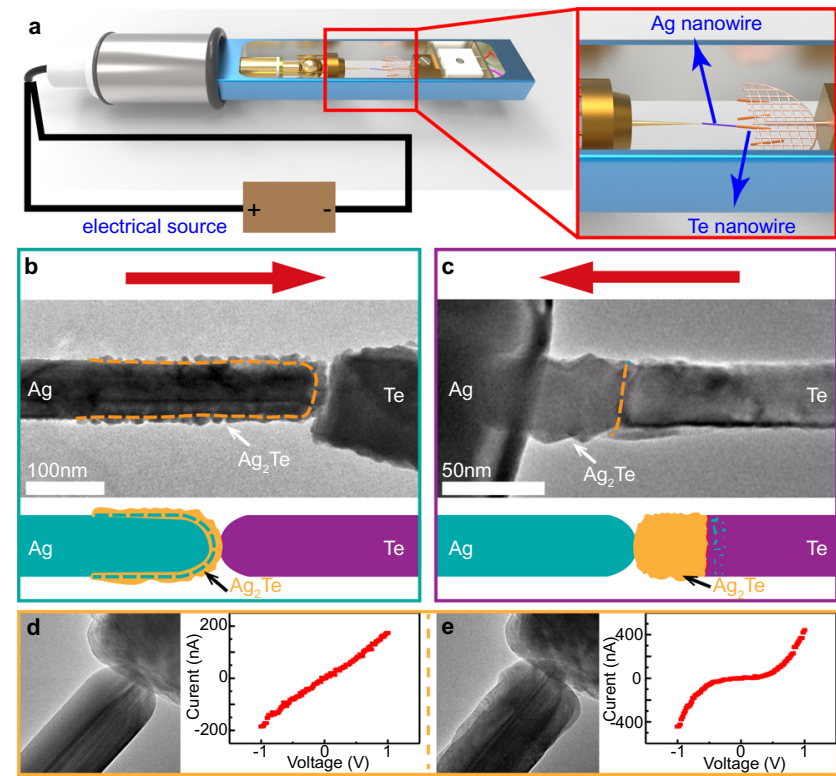

Fig. 1 The formation of 1D heterostructures by applying a bias in TEM. a Schematic diagram of the setup for in situ experiments. $\mathbf{b}$ The formation of the $\mathrm{Ag}_{2} \mathrm{Te}-\mathrm{Ag}$ core-shell structure by applying a positive voltage (red arrow showing the direction of current) to the Ag NW against the Te NW; the structure of the core-shell NW is illustrated at the bottom. c Lowmagnification TEM image and the illustration showing the formation of $\mathrm{Ag}_{2} \mathrm{Te}$-Te binary heterostructure when applying a negative voltage. $\mathbf{d} \mathrm{I}-\mathrm{V}$ curve with the corresponding TEM image of the $\mathrm{Ag}$-Te structure showing characteristics of an Ohmic contact before biasing; e I-V curve with the corresponding TEM image of the $\mathrm{Ag}_{2} \mathrm{Te}-\mathrm{Ag}$ structure showing semiconductor characteristic after biasing. 


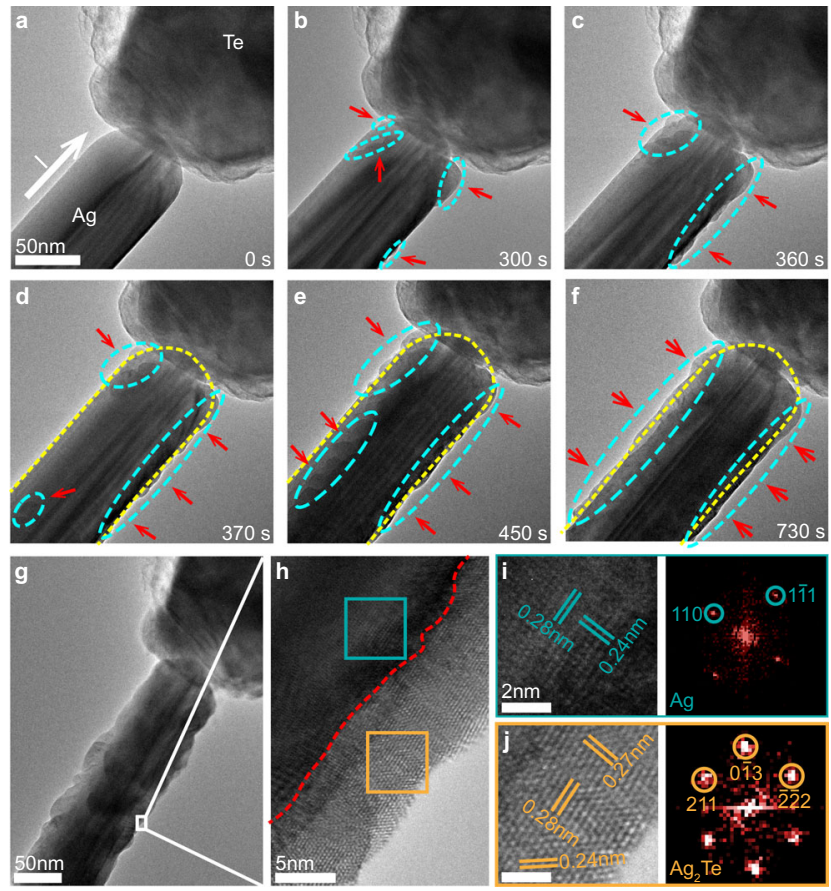

Fig. 2 Microstructure and morphology evolution of an Ag NW under positive bias. a-f TEM image series showing the formation of an $\mathrm{Ag}_{2} \mathrm{Te}-\mathrm{Ag}$ core-shell structure when $0.3 \mathrm{~V}$ bias was applied to the system. The red arrows and blue dashed circles indicate the growth of the $\mathrm{Ag}_{2} \mathrm{Te}$ crystallite or shell, whereas the yellow dotted lines trace the shape of the Ag NW before biasing. $\mathbf{g}$ Low-magnification TEM image showing the final $\mathrm{Ag}_{2} \mathrm{Te}-\mathrm{Ag}$ heterostructure. $\mathbf{h}$ High-resolution TEM image of the area enclosed by the white square in $\mathbf{g}$ with the red dashed line highlighting the boundary between the $\mathrm{Ag}$ core and the $\mathrm{Ag}_{2} \mathrm{Te}$ shell. $\mathbf{i}, \mathbf{j}$ HRTEM images (left) and the corresponding FFT patterns (right) acquired from the core and the shell respectively.

Fourier transform (FFT) patterns were analyzed to determine the structure and composition of the products. Our results confirm that the core is made of Ag and the shell has the composition $\mathrm{Ag}_{2} \mathrm{Te}$ (Fig. $2 \mathrm{~g}-\mathrm{j}$ ) with the interface along with the red dashed line. The total reaction in this experiment can be expressed by the Eq. (1):

$$
2 \mathrm{Ag}+\mathrm{Te} \rightarrow \mathrm{Ag}_{2} \mathrm{Te}
$$

Negative Gibbs free energy $\left(\triangle G_{\mathrm{f}}^{0}=-45.4 \mathrm{~kJ} \mathrm{~mol}^{-1}\right)^{25}$ in this equation indicates that the reaction is spontaneous ${ }^{26}$. Owing to the low reaction barrier, $\mathrm{Ag}$ atoms could easily react with the diffused Te atoms, resulting in the formation of $\mathrm{Ag}_{2} \mathrm{Te}$ crystals.

To investigate the process of formation of an $\mathrm{Ag}_{2} \mathrm{Te}$ shell on the atomic scale, a series of HRTEM images were collected. Figure 3a-1 shows the formation process of the $\mathrm{Ag}_{2} \mathrm{Te}$ shell including the two stages described above (Supplementary Movie 2): nucleation and growth of small hill-like structures (Fig. 3a-f), and coalescence of hillock the hill-like structures and thickening (Fig. 3g-l), which is consistent with Fig. 2. Because of the electron-wind force and the low activation energy of surface diffusion, Te atoms are expected to diffuse into the Ag NW along the surface and congregate at areas where structural defects are present (Fig. 3b). Crystals of $\mathrm{Ag}_{2} \mathrm{Te}$ nuclei form and grow into larger hill-like structures (Fig. 3c-f). The images suggest that the axial growth rate is much higher than the radial growth rate, which may be due to the fact that there is a sufficient number of $\mathrm{Te}$ atoms migrate from the Te NW on the surface and these react easily with the $\mathrm{Ag}$ NW. Adjacent $\mathrm{Ag}_{2} \mathrm{Te}$ hillocks grow and connect to each other, as shown in Fig. 3g-l, resulting in the formation of a stable shell. It is worth noting that there are more steps at the junction of two hillocks than in the middle of the individual crystallites. The greater number of steps and the faster growth can allow the hillocks to eliminate stress and connect into a stable shell structure (Fig. 3k), following which, the shell continues to thicken through a layer-by-layer growth (Fig. 31). Moreover, the growth rate of the layer is closely related to the current and the nature of the contact. When a stable contact is obtained, the growth of the $\mathrm{Ag}_{2} \mathrm{Te}$ shell is linear with time (growth rate is $\sim 0.038 \mathrm{~nm} / \mathrm{s}$ ) under a constant bias (Supplementary Fig. 2), indicating that the preparation of these structures can be tailored by changing the applied bias under certain conditions. Our experimental results also reveal the relationship between the crystallographic orientations of the $\mathrm{Ag}_{2} \mathrm{Te}-\mathrm{Ag}$ core-shell $\mathrm{NW}$ (Fig. $3 \mathrm{~m}$ ), which corresponds to the structure in Fig. 31. We identify that the orientation relationship is $\mathrm{Ag}_{2} \mathrm{Te}\{\overline{1} 23\}-\mathrm{Ag}\{111\}$ due to the matching extent of the lattices (Supplementary Fig. 3).

In order to determine the extent of surface diffusion of Te, we performed energy-dispersive X-ray spectroscopy (EDX) analysis (Fig. 3n and Supplementary Fig. 4) in STEM mode. Figure 3n shows the EDX mapping of the front part of the core-shell structured NW; results confirm the distribution of $\mathrm{Ag}$ and Te elements. The intensity profile of Te along the radial direction is uniformly flat, whereas that of Ag shows a Gaussian distribution (Fig. 3o). This suggests that the Te element is only present in the uniform shell around the NW, which further demonstrates that the migration of Te into the $\mathrm{Ag}$ NW is predominantly through surface diffusion.

Formation of segmented heterostructures. It is rather remarkable that if a negative bias is applied, as shown in Fig. 4 (Supplementary Movies 3 and 4), it is not a core-shell but a segmented binary structure that is formed. In this case, Ag atoms migrate into the Te NW driven by the electron-wind force. In the initial stage, there is no obvious structural transformation, but a slight change, in contrast, is observed on the Te NW (Fig. 4a-c), indicating the diffusion of $\mathrm{Ag}$ atoms into the Te NW. After $80 \mathrm{~s}$, structure clear phase transformation occurs (Fig. 4d and Supplementary Fig. 5), which can be identified by size changes as well as shape transformation, resulting from volume expansion accompanying the formation of $\mathrm{Ag}_{2} \mathrm{Te}$ from $\mathrm{Te}$. As time progresses, more and more $\mathrm{Ag}_{2} \mathrm{Te}$ crystals are generated, and the change in size is much evident (Fig. $4 \mathrm{~d}-\mathrm{f}$ ) with the diameter increasing by $15 \%$, which is equivalent to the $30 \%$ increase in volume during structure change from Te to $\mathrm{Ag}_{2} \mathrm{Te}$. Results from the quantitative analysis on the formation process of the diffusion rate of reaction frontier and transformation rate of $\mathrm{Ag}_{2} \mathrm{Te}$ as a function of reaction time are given in Supplementary Fig. 6. Similar to the generation process of the core-shell structure, the $\mathrm{Ag}_{2} \mathrm{Te}$ phase transformation exhibits an approximately uniform velocity $(\sim 2 \mathrm{~nm} / \mathrm{s})$ with the constant current. However, the diffusion of Ag atoms becomes progressively slower with time (from $\sim 8 \mathrm{~nm} / \mathrm{s}$ to $\sim 3 \mathrm{~nm} / \mathrm{s}$ ), which may be owing to the longer diffusion path. Notwithstanding, our results indicate that the rate of formation of the heterostructure can be tailored by changing the applied bias.

The chemical composition and structure of the reaction product were confirmed by HRTEM. As shown in Fig. 4g, there are three distinct areas in the as-formed heterostructure. The front part that is connected to the Ag NW has completely converted to $\mathrm{Ag}_{2} \mathrm{Te}$, whereas the part at the end still shows the crystal lattice of Te. It is noted here that there is a transition region between the two parts where the wire maintains the 

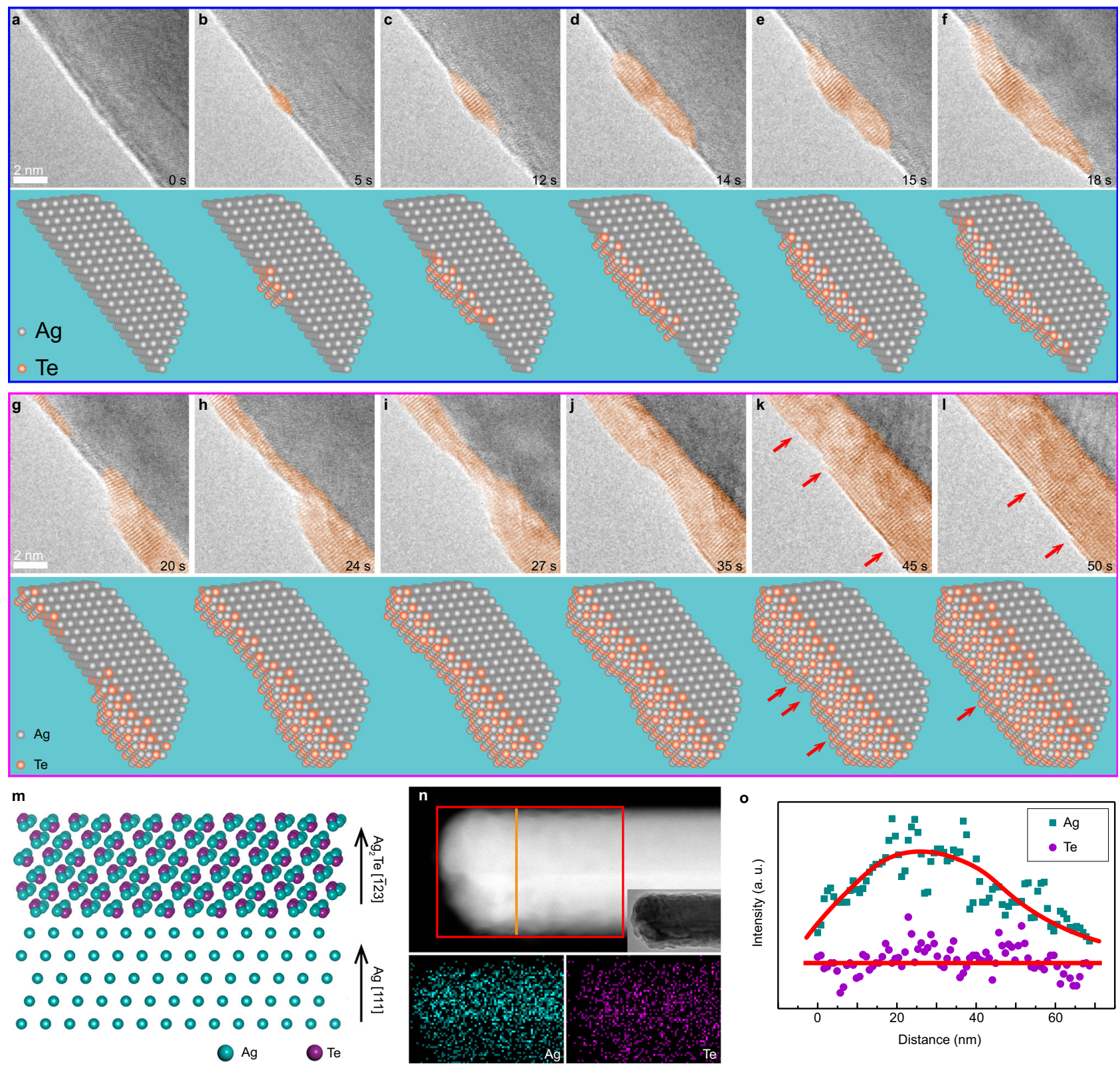

Fig. 3 The growth process of $\mathbf{A g}_{\mathbf{2}}$ Te shell. a-I TEM image series showing the growth of an $\mathrm{Ag}_{2} \mathrm{Te}$ shell consisting of two distinct stages: nucleation and growth of small hillocks a-f and hillock coalescence and thickening $\mathbf{g}-\mathbf{I}$. The red arrows highlight the growth steps of the $\mathrm{Ag}_{2} \mathrm{Te}$ layer. Below the TEM images are the corresponding structure illustrations. $\mathbf{m}$ Schematic diagram of the $\mathrm{Ag}_{2} \mathrm{Te}-\mathrm{Ag}$ heterostructure. $\mathbf{n}$ EDX elemental mapping images of $\mathrm{Ag}$ and Te corresponding to the red square area in the high angle annular dark-field (HAADF) image. o Intensity profiles of $\mathrm{Ag}$ and $\mathrm{Te}$ along the radial direction shown by the orange line in $\mathbf{n}$.

original shape and the Te lattice framework although some new structures different from that of $\mathrm{Te}$ or $\mathrm{Ag}_{2} \mathrm{Te}$ are seen. This indicates that the formation of $\mathrm{Ag}_{2} \mathrm{Te}$ can be explained on the basis of atomistic bulk diffusion and chemical phase transformation. Under the influence of electric current, Ag atoms migrate into Te NW, after which the $\mathrm{Ag}_{2} \mathrm{Te}$ phase transformation takes place when the diffused $\mathrm{Ag}$ atoms are sufficient. This explains why no obvious changes in shape and in size are seen in the initial stages of the reaction.

HAADF and EDX mapping (Fig. 4h) were obtained to analyze the chemical composition and elemental distribution in the $\mathrm{Ag}_{2} \mathrm{Te}-\mathrm{Te}$ heterostructure. It is noted that $\mathrm{Ag}$-doped Te NW can easily break in areas near the phase transformation end owing to the low thermal evaporation temperature of $\mathrm{Te}\left(<300^{\circ} \mathrm{C}\right)^{27}$. Our selected area is therefore the transition zone where a lot of Ag atoms have diffused into Te NW but have not yet completely converted to $\mathrm{Ag}_{2} \mathrm{Te}$, which means that this area is still in the diffusion stage (stage 1) of the solid-solid reaction. The intensity profile of both $\mathrm{Ag}$ and $\mathrm{Te}$ along the radial direction (Fig. 4i) presents a Gaussian distribution, indicating that the diffusion of $\mathrm{Ag}$ into Te is predominantly a bulk diffusion process. Moreover, a weak signal of the Te element was captured at a point in the front portion of the $\mathrm{Ag} \mathrm{NW}$, even though the atomic ratio of $\mathrm{Te}$ to $\mathrm{Ag}$ was determined to be only 1: $(73.31 \pm 35.27)$. (Supplementary Fig. 7). These results reveal that some thermal diffusion also occurs in the system. However, the electrically driven atomic diffusion evidently plays a major role in the transformation processes taking place in the Ag-Te system. 

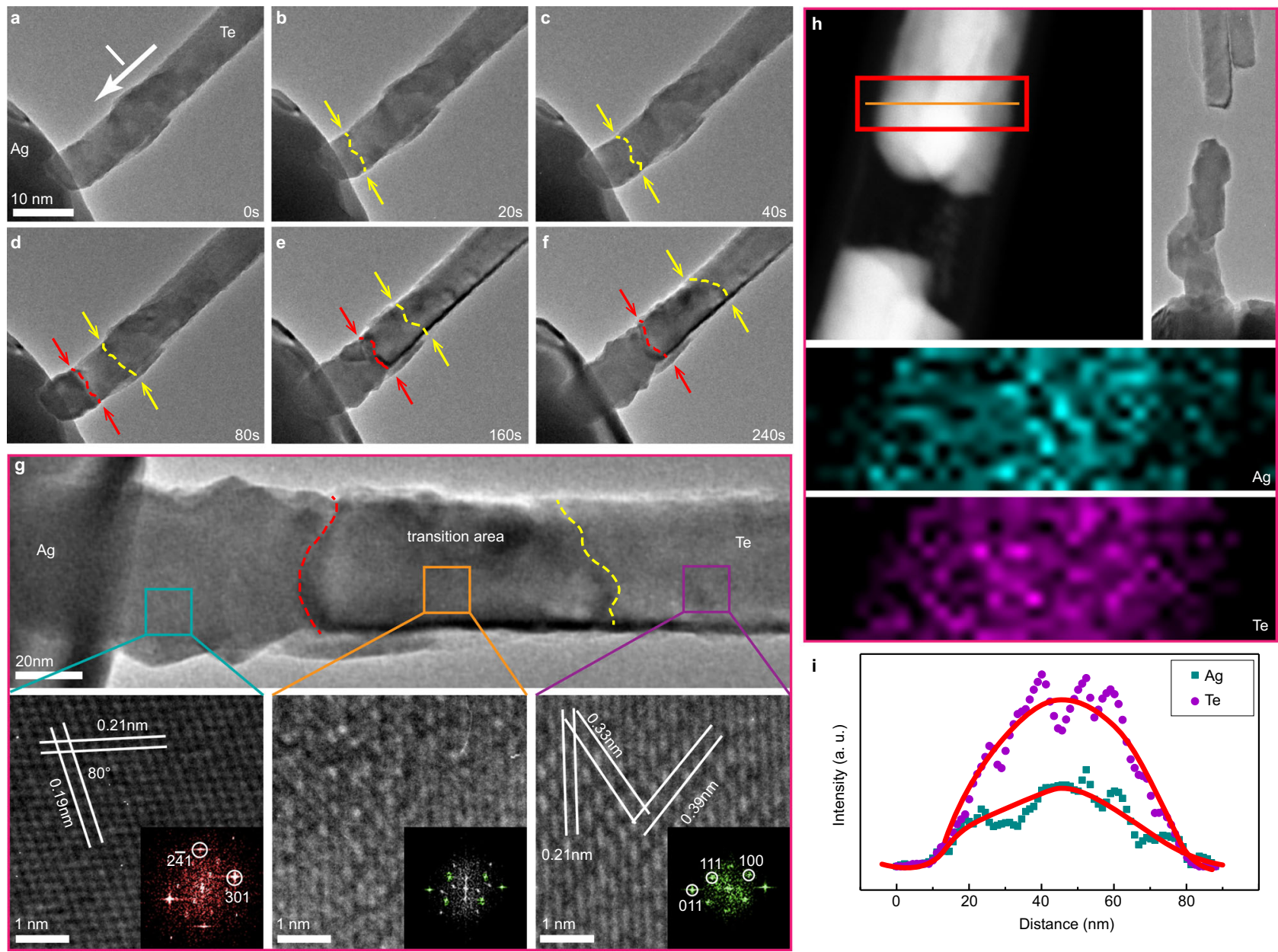

Fig. 4 The formation of an $\mathbf{A g}_{\mathbf{2}} \mathbf{T e}-\mathrm{Te}$ binary heterostructure $\mathbf{N W}$. a-f $\mathrm{TEM}$ image series showing the growth of $\mathrm{Ag}_{2} \mathrm{Te}$ under $-0.8 \mathrm{~V}$ bias applied to an $\mathrm{Ag}$ NW. The yellow dashed lines highlight the diffusion frontier, and the red dashed lines indicate the phase transformation frontier. $\mathbf{g}$ TEM image showing the fully formed $\mathrm{Ag}_{2} \mathrm{Te}-\mathrm{Te}$ heterostructure in the Te NW. Shown at the bottom are HRTEM images and the corresponding FFT images acquired from different areas on the $\mathrm{Ag}_{2} \mathrm{Te}-\mathrm{Te} \mathrm{NW}$. $\mathbf{h}$ EDX elemental mapping images of $\mathrm{Ag}$ and Te corresponding to the red square area in the HAADF image. $\mathbf{i}$ Intensity profiles of $\mathrm{Ag}$ and $\mathrm{Te}$ showing the elemental distribution in the radial direction along the orange line in $\mathbf{h}$.

\section{Discussion}

Although electron beam irradiation may trigger atomic diffusion $^{28-30}$, the diffusion is not predominated by an electron beam in this work. Control experiments demonstrated that no obvious changes occur either in Ag or Te NWs even if the contacted system is exposed to electron beam without electrical bias for a long time (Supplementary Fig. 8). They are clearly different from the previous report that $\mathrm{Ag}_{2} \mathrm{Te} \mathrm{NW}$ could be formed only under electron beam irradiation when $\mathrm{Ag}$ and Te NWs were placed on the support film in close proximity ${ }^{30}$. The contrary phenomena should result from the different diffusion paths. When Ag and Te NWs are placed on a film, Ag species excited by electron beam irradiation could migrate in all directions on the film surface. In our experiments, Ag and Te NWs are suspended and point contacted, the excited species can only diffuse through the contact region, which will seriously limit the atomic diffusion and the formation of $\mathrm{Ag}_{2} \mathrm{Te}$. On the other hand, tens of experiments presented similar phenomena (Supplementary Figs. 9-11) that the atomic diffusion is strongly dependent on the direction of the electric field. However, electron beam-induced atomic diffusion should be isotropic, which indicates that the influence of electron beam can be ignored, and the diffusion should be driven by an electric field.

When a bias voltage is applied to Ag-Te NWs through the contact, collisions between the conduction electrons and the atoms can lead to the migration of the atoms. The driving force of electromigration is shown in Fig. 5a and Supplementary Fig. 12. For $\mathrm{Ag}$ and $\mathrm{Te}$ in this work, the atoms migrate in the direction opposite to the electric field ${ }^{31,32}$. When a negative voltage is applied to Te NW, Te atoms migrate into the Ag NW. Although there are other diffusion mechanisms (thermal diffusion or concentration gradient diffusion, Supplementary Fig. 7), we stress that electromigration has a significant role in this process because the diffusion direction is strongly dependent on the applied bias.

It is important to emphasize that the process observed in this work is not an electrochemical process. As reported in our previous work $^{19}$, electrochemical cation exchange reaction only occurs if the applied voltage is larger than the standard electrode potential of the material. In this work, atomic diffusion occurred even if the applied voltage $(<0.5 \mathrm{~V})$ is far lower than the corresponding standard electrode potential ${ }^{33}, E^{0}\left(\mathrm{Ag}^{+} / \mathrm{Ag}\right)=0.799 \mathrm{~V}$, $E^{0}\left(\mathrm{Te} / \mathrm{Te}^{2-}\right)=-1.143 \mathrm{~V}$, when both $\mathrm{Ag}$ and $\mathrm{Te}$ cannot be ionized electrochemically (Supplementary Fig. 13). It is also noted that the directions of atomic or ions diffusion are totally different. The direction of $\mathrm{Cu}^{+}$diffusion driven by the electrostatic force in the electrochemical process is consistent with the direction of the electric field ${ }^{19}$, whereas, in this work, the direction of atomic diffusion of $\mathrm{Ag}$ or Te atoms is completely opposite to the direction of electric field, as the driving force of the atomic diffusion is electron-wind force. Therefore, we consider that the process is 


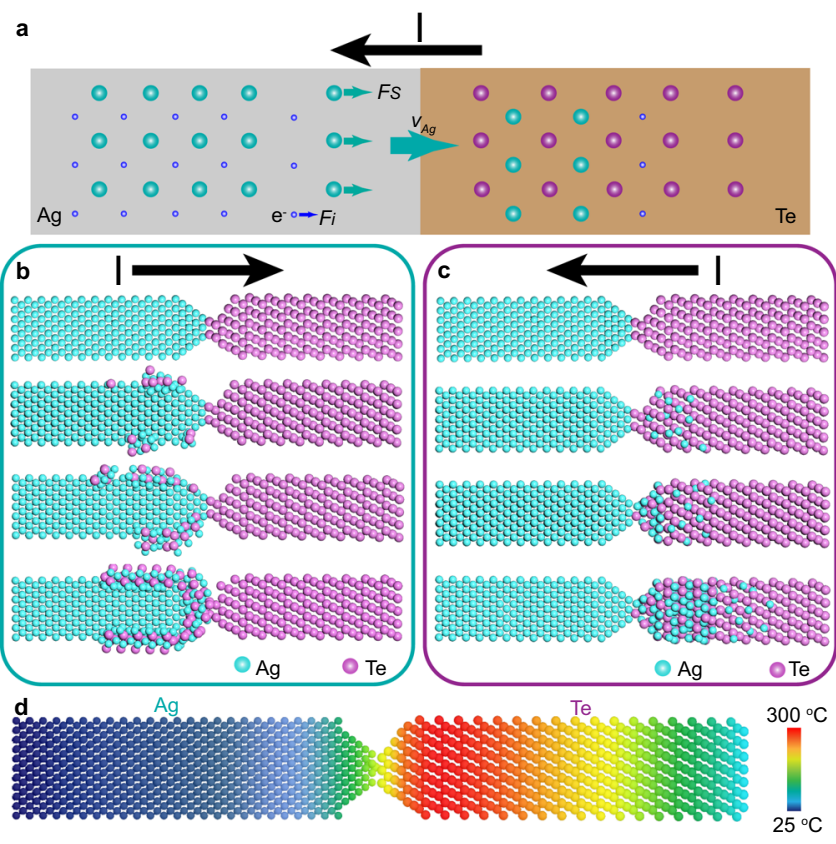

Fig. 5 Formation mechanism of Ag-Te heterostructure. a Schematic

illustration of the forces acting on the Ag-Te system under a negative bias voltage. $F_{i}$ and $F_{s}$ separately indicate electrostatic forces and the ion or atom scattering forces (electron-wind force). b, c Schematic diagrams showing the formation processes of $\mathrm{Ag}_{2} \mathrm{Te}-\mathrm{Ag}$ core-shell structured NW and $\mathrm{Ag}_{2} \mathrm{Te}-\mathrm{Te}$ structured NW. d Schematic image showing the temperature distribution in the $\mathrm{Ag}-\mathrm{Te}$ system.

electromigration-dominated diffusion followed by the spontaneous chemical reaction between $\mathrm{Ag}$ and $\mathrm{Te}^{19,22}$.

As shown in Supplementary Figs. 10 and 11, no matter what conditions were, similar phenomena were observed and can be summarized in Fig. 5b, c. When a positive voltage is applied to the Ag-Te system, Te atoms diffuse into Ag NW along the surface under the influence of electron-wind force, and then the migrated Te atoms react with $\mathrm{Ag}$ to form $\mathrm{Ag}_{2} \mathrm{Te}$ crystallite. Small pieces of $\mathrm{Ag}_{2} \mathrm{Te}$ islands are connected into sheets, and finally, a shell layer is formed on the surface of the Ag NW (Fig. 5b). In contrast, Ag atoms begin to migrate into Te crystal by the path of bulk diffusion under a negative voltage bias. As shown in Fig. $5 \mathrm{c}, \mathrm{Te}$ atoms and the migrated $\mathrm{Ag}$ atoms will undergo a process of phase transformation to form $\mathrm{Ag}_{2} \mathrm{Te}$ when the atomic ratio is appropriate. Finally, the Te NW becomes an $\mathrm{Ag}_{2} \mathrm{Te}$-Te binary heterostructure. However, the current density might have an influence on the reaction speed. The reaction of thinner nanowires is faster than that of the thicker nanowires (Supplementary Fig. 10c, d, Fig. 2 and Supplementary Fig. 10b). It should be noticed that the formation of heterostructure is limited by the contact conditions, but it is hard to ensure the same contact in the separate experiment and the contact conditions are difficult to be accurately quantified inside the microscope, which makes challenges to reveal quantitative voltage-dependent kinetics from various Ag-Te systems (Supplementary Fig. 14).

The diffusion path may affect the final morphology of the asformed heterostructures. Generally, surface diffusion takes priority over bulk diffusion in the solid materials owing to the lower diffusion barrier, however, bulk diffusion can be comparable if the system temperature is high enough when the thermal energy could help more atoms or ions to conquer the barrier of bulk diffusion.

We stress that temperature has a significant role in our process, and crucially affects the diffusion path, and eventually results in the formation of heterostructures with entirely different morphologies ${ }^{34-37}$. We constructed a model to probe the temperature distribution of the Ag-Te 1D system under a bias (Supplementary Fig. 15 and Supplementary Note 1). When a voltage bias is applied to the $1 \mathrm{D}$ conductor system, heat will be generated along the conductor via Joule heating. Considering that the heat loss by radiative processes is negligible in vacuum (in a TEM), the heat can only be dissipated along the two contacted conductors through the contacts at their ends. The 1D conductor system can be set up to the state of thermal equilibrium within a short time $\mathrm{e}^{38}$.

For simplicity of calculation, we first consider the condition where the contact resistance is zero for the system. According to the general heat conduction equation, the maximum temperature rise by Joule heating can be estimated by ${ }^{38}$

$$
\triangle T=T_{\max }-T_{0}=\frac{\sigma U^{2}}{8 \kappa}
$$

where $U$ is the voltage drop on the NW, $T_{0}$ is the temperature of contact electrodes (room temperature). $\sigma$ and $\kappa$ are electrical conductivity and thermal conductivity respectively, which are assumed to be constant. It should be noticed that the contact resistance between $\mathrm{Ag}$ and Te nanowire is typically large owing to the amorphous carbon layer and could not be neglected. Depending on various conditions of contact, the contact resistance may range from $10^{4}$ to $10^{9} \Omega^{39,40}$ and the heat generated from that will be large due to Joule heating. In consideration of the large difference of thermal conductivity between $\mathrm{Ag}\left(429 \mathrm{~W} \mathrm{~m}^{-1} \mathrm{~K}^{-1}\right)$ and $\mathrm{Te}\left(2.35 \mathrm{~W} \mathrm{~m}^{-1} \mathrm{~K}^{-1}\right)$, the side of Te NW will show a much higher and slowly changing temperature while the temperature reduces to the room temperature in a very short distance in $\mathrm{Ag}$ NW, as shown in Fig. 5d.

Applying typical parameters ${ }^{27}$ of Te to Eq. (2), the maximum temperature rise of Te NW can be estimated to be over $300 \mathrm{~K}$, which is close to the melting temperature of $\mathrm{Te}^{41}$. If the Umklapp phonon-phonon scattering process and contact resistance are considered, the maximum temperature $T_{\max }$ is expected to be higher $^{42}$. Meanwhile, owing to the low thermal conductivity of Te, the heat generated by the contact resistance can promote the temperature of Te side when the heat is conducted along the $\mathrm{Te}$ NW. Once the Ag atoms diffuse into Te NW owing to the electromigration, the large thermal energy on the Te NW could be enough for Ag atoms to conquer the barrier of bulk diffusion in Te. Consequently, bulk diffusion is substantial for Ag atoms in Te $\mathrm{NW}$, resulting in the formation of binary hetero-structured NW. In contrast, use typical parameters for $\mathrm{Ag}$, we can estimate the maximum temperature rise at $\mathrm{Ag} \mathrm{NW}$ to be $<10^{-2} \sim 10 \mathrm{~K}^{43}$. For the diffused Te atoms in Ag NW, surface diffusion still dominates over bulk diffusion due to the low barrier of surface diffusion, resulting in the nucleation and growth of a shell on the surface of Ag NW. Notably, the temperature distribution of the system is the same when the direction of current changes.

Following this analysis, this strategy can also be carried out in other materials. The direction of atomic diffusion can be tailored by current, whereas the heat generated by Joule heating can alter the path of atomic diffusion. Although other methods can produce well-defined heterostructures ${ }^{44}$, selective customization for individual nanostructures still remains challenging. Using this method, controlled selective synthesis of individual heterostructure could be achieved by simply adjusting the current and local temperature, which is critical to tailor nanostructures and for the construction of future nanodevices.

In summary, an electrically driven solid-solid diffusionreaction in the Ag-Te NW system was investigated using in situ TEM. The atomic diffusion process can be tailored by voltage bias in order to selectively synthesize individual hetero- 
nanostructured NWs. The reaction mechanism is strongly supported by the fact that the diffused atoms are mainly driven by electron-wind force along the direction electrons flowed, whereas the phase transformation occurs spontaneously with an appropriate atomic ratio of $\mathrm{Ag}$ and $\mathrm{Te}$. Moreover, the diffusion path can be tailored by local temperature caused by Joule heating, resulting in the formation of heterostructures with distinct morphologies. Surface diffusion of Te atoms in Ag NWs leads to the core-shell structure, whereas bulk diffusion of $\mathrm{Ag}$ atoms in $\mathrm{Te}$ NWs results in a segmented binary hetero-structured NW. Our results provide a new perspective to synthesis individual nanostructures with a diffusion-controlled reaction and also throw light on the micro-mechanism of solid-solid processes.

\section{Methods}

Preparation of Ag NW and Te NW. Both Ag and Te NW samples were prepared by a solvothermal chemical approach. Ag NW preparation: $10 \mathrm{~mL}$ of ethylene glycol (EG, AR, 98\%, Aladdin) and $1.5 \mathrm{~g}$ of polyvinyl pyrrolidone (PVP, molecule weight $\approx 24,000$, Aladdin) was injected dropwise into $10 \mathrm{~mL}$ of a magnetically stirred solution of $0.1 \mathrm{M}$ silver nitrate $\left(\mathrm{AgNO}_{3}, \mathrm{AR}, 99.8 \%\right.$, Aladdin). The solution was then transferred to a Teflon-lined autoclave tube. The tube was sealed and heated at $160^{\circ} \mathrm{C}$ for $2.5 \mathrm{~h}$. The reaction mixture was cooled naturally to room temperature and the products were washed several times with acetone and water. Te NW preparation: $0.5 \mathrm{~g}$ tellurium dioxide $\left(\mathrm{TeO}_{2}, 99.99 \%\right.$, Aladdin), $1.5 \mathrm{~g}$ PVP (molecule weight $\approx 24,000$, Aladdin), $7.5 \mathrm{~mL} \mathrm{NH} \mathrm{H}_{3} \cdot \mathrm{H}_{2} \mathrm{O}$ (ACS, 28.0-30.0\%, Aladdin) and $1.625 \mathrm{~mL}$ hydrazine monohydrate $(>98.0 \%(\mathrm{~T})$, Aladdin) were dissolved in $110 \mathrm{~mL}$ deionized water under vigorous stirring and then transferred to a Teflon-lined stainless steel autoclave. The sealed autoclave was maintained at $200{ }^{\circ} \mathrm{C}$ for $16 \mathrm{~h}$, followed by cooling naturally to room temperature. The products were washed five times alternately with ethanol (ACS, Aladdin) and water.

In situ TEM experiment. A half $\mathrm{Cu}$ grid, onto which a drop of a solution of asprepared Te NWs was placed, was glued onto a gold wire using conductive epoxy. During this process, some Te NWs were anchored at the edge of the half $\mathrm{Cu}$ grid. W tips were prepared by electrochemically etching with $\mathrm{NaOH}$ solution $(2 \mathrm{M})$ under a voltage of $2 \mathrm{~V}$. All the $\mathrm{W}$ tips were cleaned by plasma cleaning for $60 \mathrm{~s}$ before use. The $\mathrm{W}$ tips were then soaked in the solution of as-prepared Ag NWs for $60 \mathrm{~s}$ at the end of which, several Ag NWs adhered to the surface of the tip. The setup of the in situ electrical experiments is shown in Fig. 1a. All the in situ experiments in this work were conducted in vacuum.

Characterization of the structures. The NWs and as-prepared hetero-nanostructures were characterized and analyzed using an aberration-corrected TEM (Titan 80-300, FEI). The electrical property measurements were carried out using a TEM-STM holder (PicoFemto). Dark-field images and EDX mapping were performed in STEM mode.

\section{Data availability}

All the data supporting the findings of this study are available within this article and its Supplementary Information files. All raw images and source data are available from the authors upon reasonable request.

Received: 28 May 2020; Accepted: 23 July 2021;

Published online: 10 August 2021

\section{References}

1. Tan, C. \& Zhang, H. Epitaxial growth of hetero-nanostructures based on ultrathin two-dimensional nanosheets. J. Am. Chem. Soc. 137, 12162-12174 (2015).

2. Kronawitter, C. X. et al. A perspective on solar-driven water splitting with alloxide hetero-nanostructures. Energy Environ. Sci. 4, 3889 (2011).

3. Zhang, J., Tang, Y., Lee, K. \& Ouyang, M. Tailoring light-matter-spin interactions in colloidal hetero-nanostructures. Nature 466, 91 (2010).

4. Mai, L., Sheng, J., Xu, L., Tan, S. \& Meng, J. One-dimensional heteronanostructures for rechargeable batteries. Acc. Chem. Res. 51, 950-959 (2018).

5. Xiong, C., Aliev, A. E., Gnade, B. \& Balkus, K. J. Jr Fabrication of silver vanadium oxide and $\mathrm{V}_{2} \mathrm{O}_{5}$ nanowires for electrochromics. ACS Nano. 2 , 293-301 (2008).

6. Xu, H. et al. Dynamic structure-properties characterization and manipulation in advanced nanodevices. Mater. Today Nano 7, 100042 (2019).
7. Sheldon, M. T., Trudeau, P., Mokari, T., Wang, L. \& Alivisatos, A. P. Enhanced semiconductor nanocrystal conductance via solution grown contacts. Nano Lett. 9, 3676-3682 (2009).

8. Zhang, $\mathrm{M}$. et al. One-dimensional $\mathrm{Bi}_{2} \mathrm{MoO}_{6} / \mathrm{TiO}_{2}$ hierarchical heterostructures with enhanced photocatalytic activity. Crystengcomm 14, 605-612 (2012).

9. Wang, W. et al. Edge-terminated few-layer $\mathrm{MoS}_{2}$ nanoflakes supported on TNAs@C with enhanced electrocatalysis activity for iodine reduction reaction. Mater. Today Nano 6, 100033 (2019).

10. Lo, S. S., Mirkovic, T., Chuang, C., Burda, C. \& Scholes, G. D. Emergent properties resulting from type-II band alignment in semiconductor nanoheterostructures. Adv. Mater. 23, 180-197 (2011).

11. Li, Z., Song, P., Yang, Z. \& Wang, Q. In situ formation of one-dimensional $\mathrm{CoMoO}_{4} / \mathrm{MoO}_{3}$ heterojunction as an effective trimethylamine gas sensor. Ceram. Int. 44, 3364-3370 (2018).

12. Seifert, W. et al. Growth of one-dimensional nanostructures in MOVPE. J. Cryst. Growth 272, 211-220 (2004).

13. Mieszawska, A. J., Jalilian, R., Sumanasekera, G. U. \& Zamborini, F. P. The synthesis and fabrication of one-dimensional nanoscale heterojunctions. Small 3, 722-756 (2007)

14. Tian, B. et al. Single-crystalline kinked semiconductor nanowire superstructures. Nat. Nanotechnol. 4, 824 (2009).

15. Lauhon, L. J., Gudiksen, M. S., Wang, D. \& Lieber, C. M. Epitaxial core-shell and core-multishell nanowire heterostructures. Nature 420, 57 (2002).

16. Yang, G., Yan, W., Zhang, Q., Shen, S. \& Ding, S. One-dimensional CdS/ZnO core/shell nanofibers via single-spinneret electrospinning: tunable morphology and efficient photocatalytic hydrogen production. Nanoscale 5, 12432-12439 (2013).

17. Chen, Y. et al. Porous iron molybdate nanorods: in situ diffusion synthesis and low-temperature $\mathrm{H}_{2} \mathrm{~S}$ gas sensing. ACS Appl. Mater. Inter. 5, 3267-3274 (2013).

18. Resasco, J. et al. $\mathrm{TiO}_{2} / \mathrm{BiVO}_{4}$ Nanowire heterostructure photoanodes based on type II band alignment. ACS Cent. Sci. 2, 80-88 (2016).

19. Zhang, Q. et al. Electrically driven cation exchange for in situ fabrication of individual nanostructures. Nat. Commun. 8, 14889 (2017).

20. Ho, P. S. \& Kwok, T. Electromigration in metals. Rep. Prog. Phys. 52, 301-348 (1989).

21. He, L. et al. Novel behaviors/properties of nanometals induced by surface effects. Mater. Today Nano 1, 8-21 (2018).

22. Mei, S. et al. Dynamic investigation of interface atom migration during heterostructure nanojoining. Nanoscale 6, 405-411 (2014).

23. Chen, D. et al. Large-scale synthesis of silver nanowires via a solvothermal method. J. Mater. Sci. Mater. Electron. 22, 6-13 (2011).

24. Lu, Q., Gao, F. \& Komarneni, S. A Green chemical approach to the synthesis of tellurium nanowires. Langmuir 21, 6002-6005 (2005).

25. Karakaya, I. \& Thompson, W. T. The Ag-Te (silver-tellurium) system. J. Phase Equilibria 12, 56-63 (1991).

26. Zhang, M. et al. Thermoelectric properties of ultralong silver telluride hollow nanofibers. Chem. Mater. 27, 5189-5197 (2015).

27. Cotton, A. F., Wilkinson, G., Bochmann, M. \& Murillo, C. A. Advanced inorganic chemistry. Vol. 6. (Wiley, New York, 1988).

28. Zheng, H. et al. Controlling electron beam-induced structure modifications and cation exchange in cadmium sulfide-copper sulfide heterostructured nanorods. Ultramicroscopy 134, 207-213 (2013).

29. Motte, L. \& Urban, J. Silver clusters on silver sulfide nanocrystals: synthesis and behavior after electron beam irradiation. J. Phys. Chem. B. 109, 21499-21501 (2005)

30. He, Z. et al. Real-time visualization of solid-phase ion migration kinetics on nanowire monolayer. J. Am. Chem. Soc. 142, 7968-7975 (2020)

31. Orlov, A. M. \& Skvortsov, A. A. Temperature gradient effect on the electromigration of Ag-Te melt inclusions in tellurium. Inorg. Mater. 43 471-474 (2007).

32. Hauder, M., Gstöttner, J., Hansch, W. \& Schmitt-Landsiedel, D. Scaling properties and electromigration resistance of sputtered Ag metallization lines. Appl. Phys. Lett. 78, 838-840 (2001)

33. Speight, J. G. Lange's Handbook of Chemistry, 70th Anniversary Edition. (McGraw-Hill Professional Publishing, New York, 2004).

34. Surholt, T., Minkwitz, C. \& Herzig, C. Solute diffusion and segregation in grain boundaries of silver and copper. Defect Diffus. Forum 156, 59-74 (1998).

35. Seebauer, E. G. \& Allen, C. E. Estimating surface diffusion coefficients. Prog. Surf Sci. 49, 265-330 (1995)

36. Levine, L. E., Reiss, G. \& Smith, D. A. In situ scanning-tunneling-microscopy studies of early-stage electromigration in Ag. Phys. Rev. B. 48, 858-863 (1993).

37. Johnson, D. B. \& Brown, L. C. Lateral diffusion in Ag-Te thin film couples. J. Appl. Phys. 40, 1711-1714 (1969)

38. Zhao, J., Huang, J., Wei, F. \& Zhu, J. Mass Transportation mechanism in electric-biased carbon nanotubes. Nano Lett. 10, 4309-4315 (2010).

39. Mohney, S. E. et al. Measuring the specific contact resistance of contacts to semiconductor nanowires. Solid State Electron. 49, 227-232 (2005). 
40. Dong, L. et al. Effects of local Joule heating on the reduction of contact resistance between carbon nanotubes and metal electrodes. J. Appl. Phys. 101, 24320 (2007)

41. Deaton, B. C. \& Blum, F. A. Jr. Properties of group VI B elements under pressure. I. Melting curves of S, Se, and Te. Phys. Rev. 137, A1131 (1965).

42. Huang, X. Y., Zhang, Z. Y., Liu, Y. \& Peng, L. Analytical analysis of heat conduction in a suspended one-dimensional object. Appl. Phys. Lett. 95, 143109 (2009).

43. Zhao, J., Sun, H., Dai, S., Wang, Y. \& Zhu, J. Electrical breakdown of nanowires. Nano Lett. 11, 4647-4651 (2011).

44. Steimle, B. C. et al. Experimental insights into partial cation exchange reactions for synthesizing heterostructured metal sulfide nanocrystals. Chem. Mater. 32, 5461-5482 (2020).

\section{Acknowledgements}

This work was supported by the National Science Foundation of China (no. 61601116, 61974021,51420105003 ), the national science fund for distinguished young scholars (no. 11525415), the Natural Science Foundation of Jiangsu Province (BK20181284), and the China Scholarship Council.

\section{Author contributions}

H.Z. and T.X. conceived and designed the project. H.Z. and L.H. performed the synthesis of materials. H.Z. and T.X. performed the in situ TEM experiments. H.Z. and T.X. carried out the data analysis. H.Z., T.X., K.Y., W.W., and L.S. discussed the results. H.Z., T.X., and L. Sun wrote the manuscript.

\section{Competing interests}

The authors declare no competing interests.

\section{Additional information}

Supplementary information The online version contains supplementary material available at https://doi.org/10.1038/s41467-021-25194-2.

Correspondence and requests for materials should be addressed to T.X. or L.S.

Peer review information Nature Communications thanks Bryce Sadtler and the other, anonymous, reviewer(s) for their contribution to the peer review of this work. Peer reviewer reports are available.

Reprints and permission information is available at http://www.nature.com/reprints

Publisher's note Springer Nature remains neutral with regard to jurisdictional claims in published maps and institutional affiliations.

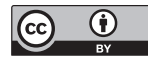

Open Access This article is licensed under a Creative Commons Attribution 4.0 International License, which permits use, sharing, adaptation, distribution and reproduction in any medium or format, as long as you give appropriate credit to the original author(s) and the source, provide a link to the Creative Commons license, and indicate if changes were made. The images or other third party material in this article are included in the article's Creative Commons license, unless indicated otherwise in a credit line to the material. If material is not included in the article's Creative Commons license and your intended use is not permitted by statutory regulation or exceeds the permitted use, you will need to obtain permission directly from the copyright holder. To view a copy of this license, visit http://creativecommons.org/ licenses/by/4.0/.

(C) The Author(s) 2021 